\title{
Nonlinear Output Regulation with Input Delay and Application in AHV Attitude Control
}

\author{
Liang Cai and Qingxian Wu \\ Automation College, Nanjing University of Aeronautics and Astronautics, Nanjing, China \\ Correspondence should be addressed to Liang Cai; caisanchun@163.com
}

Received 11 February 2017; Revised 23 March 2017; Accepted 30 March 2017; Published 8 May 2017

Academic Editor: Christopher J. Damaren

Copyright (C) 2017 Liang Cai and Qingxian Wu. This is an open access article distributed under the Creative Commons Attribution License, which permits unrestricted use, distribution, and reproduction in any medium, provided the original work is properly cited.

\begin{abstract}
Time delay phenomena exist almost everywhere in the actual world and are especially important in the control of an AHV. The small time delay usually ignored in control may cause quite obvious errors in the flight trajectory tracking, because of the extremely high flight speed. In this paper, a new framework of solving a nonlinear input delay regulation problem is proposed for the first time, which is comprised of nonlinear damping controllers, nonlinear disturbance observers, and a nonlinear time delay predictor-based compensator, under the proved separation principle. Then the attitude tracking control of an AHV with a known time-varying input delay is studied based on the proposed design framework. Simulation results show the advantage of the proposed control method.
\end{abstract}

\section{Introduction}

Tracking and disturbance rejection, usually coexisting as the aims of system control, can be treated uniformly in the output regulation problem framework [1]. This has been studied for decades [2]. For linear systems, Francis [3] illustrates that the output regulation problem could be solved based on the internal model principle. In [3], it is found that the solvability of the regulation problem is equal to that of a set of linear equations named the regulation equations. Since the 1990s, the research of output regulation of nonlinear systems has been concentrated on. In [4], Isidori and Byrnes show the equivalency between the solvability of nonlinear output regulation problems and that of a set of partial differential equations, called the regulation equations in nonlinear form or Francis-Isidori-Byrnes (FIB) equations. Recently, a general framework for output regulation problems is proposed and some general results are obtained in [5]. Depending on different research focuses, regulation problems can be divided into several types [6]: output regulation without robust [7], locally robust output regulation [8], globally robust output regulation $[9,10]$, and semiglobally robust output regulation [11]. Output regulation problems have also been developed by broadening the range of systems, not only from linear systems to nonlinear ones but also from the ones with uncertainties just in the controlled system to those with uncertainties both in the controlled system and in the exosystem, even including some other types such as time-varying systems or distributed systems $[12,13]$. When the uncertainty only exists in controlled systems, as long as an effective internal model has already been constructed [14], well-developed robust techniques, such as the small gain theory and the sliding mode control, can be used to treat the uncertainty. When there are uncertainties in the exosystem, many valuable attempts have been done in [15-17]. Dynamic high gains and selftuning regulators can be used to treat the uncertainties with unknown upper bound and parameter uncertainties in the exosystem $[18,19]$. If the direction of control is unknown, the Nussbaum gain method [20,21] is suitable to use. Treating uncertainties in the output regulation problem usually involve the estimation of unknown parameters. It is a quite important and difficult issue to assure the estimation value reaching the parameters' actual value. A research on this topic is given in [22]. Besides uncertainties, other terms, such as time delay, could also be brought into the systems.

Time delay phenomena exist almost everywhere in the actual world, such as actuator and sensor delays, but fall outside the scope of the standard finite-dimensional systems. 
In the analysis and synthesis of time delay linear systems, quite a lot of results have been given in $[23,24]$. Mainly used methods for the analysis and synthesis include character roots distribution in the frequency field [25], robust stabilization based on the small gain theory [26], the Lyapunov-Krasovskii functional theorem [27], the Lyapunov-Razumikhin function theorem [28], and the free weighting matrices technique [29] based on model transforming [30]. To treat cross-terms in the derivatives of the Lyapunov functions or functionals, several inequations are used, such as the standard inequation (Young's inequation), Park's inequation [31], Moon's inequation [32], and Jasen's integral inequation [33]. With the developing of nonlinear system theories, expanding the scope of delayed systems to nonlinear systems has aroused great interests. There are some results for specific nonlinear systems [34-37], and they are mainly focused on analysis and control design of retard delay systems. For linear systems with input delay, they can be treated by transforming the input delay into the state delay and then giving the controllers in the form of LMIs. It is much more difficult to treat nonlinear systems with input delay. For nonlinear delay systems, it is impossible to cancel out nonlinear terms using a delayed input and difficult to solve NMIs to assure the stability of the closed loop. Only recently, a few methods exist in dealing with stabilization of nonlinear systems with input delay [38-46]. Among them, the predictor-based compensation method [41-46] is particularly useful, because compensators can be well combined with controllers to achieve multiple control targets.

To our knowledge, the nonlinear output regulation problem with input time delay has been little studied. When the nonlinear regulation equations contain delay terms, it is impossible to get an analytic solution. In order to bypass solving regulation equations, observers can be used as a special kind of internal models $[47,48]$. There are only a few papers on the output regulation problem of delay systems, both for linear systems and nonlinear systems with delay $[49,50]$, and none of them deals with input delay. The main novelty in this article is a new framework of solving a nonlinear input delay regulation problem for the first time, which consists of nonlinear damping controllers, nonlinear disturbance observers, and a nonlinear time delay compensator, under the proved separation principle.

Recently, the flight control of air-breathing hypersonic vehicles (AHVs) attracts more and more research interests. There are some results on the topic, such as the flight control based on the dynamic inversion, the adaptive control, the sliding mode control, and the backstepping method $[51,52]$. Some features which are not so significant for common aircrafts become quite important for AHVs, such as nonlinearity and time delay. Small time delays usually ignored in control may cause quite obvious errors in the flight trajectory tracking, because of the extremely high flight speed. In this paper, the attitude tracking control of an AHV with input delay is studied for the first time, which is not seen elsewhere. The attitude tracking controller is derived based on the proposed design framework. The problem description is presented in the Section 2, and then the input delay compensation method is given in Section 3. In Section 4, the design of a time delay compensation controller for a class of nonlinear systems is presented and the controller's performance is summarized as theorems. Then, in Section 5, the controller is applied to the attitude control of an AHV, and simulation results show that the disturbance rejection and input time delay compensation controller for the AHV attitude tracking is effective.

\section{Problem Formulation}

Consider a class of strict feedback MIMO nonlinear system with a time-varying input delay described as below:

$$
\begin{aligned}
\dot{x}_{1}= & f_{1}\left(x_{1}\right)+g_{1}\left(x_{1}\right) x_{2}+g_{1 d}\left(x_{1}\right) d, \\
\vdots & \\
\dot{x}_{i}= & f_{i}\left(x_{1}, \ldots, x_{i}\right)+g_{i}\left(x_{1}, \ldots, x_{i}\right) x_{i+1} \\
& +g_{i d}\left(x_{1}, \ldots, x_{i}\right) d, \\
& \vdots \\
\dot{x}_{n} & =f_{n}\left(x_{1}, x_{2}, \ldots, x_{n}\right) \\
& +g_{n}\left(x_{1}, x_{2}, \ldots, x_{n}\right) u(t-D(t)) \\
& +g_{n d}\left(x_{1}, x_{2}, \ldots, x_{n}\right) d, \\
e & =x_{1}-y_{r},
\end{aligned}
$$

where the state vector $x_{i} \in R^{k}$, the whole state vector $x=$ $\left[x_{1}, \ldots, x_{n}\right] \in R^{n k}$, the input $u \in R^{k}$, a reference signal $y_{r} \in$ $R^{k}$, the time delay function $D(t)$ is accurately known, and $f_{i}\left(x_{1}, \ldots, x_{i}\right), g_{i}\left(x_{1}, \ldots, x_{i}\right)$, and $g_{i d}\left(x_{1}, \ldots, x_{i}\right)$ are all known and locally Lipschitz. The unknown disturbance $d \in R^{m}$ is produced by the autonomous exosystem

$$
\begin{aligned}
& \dot{\xi}=A \xi, \\
& d=C \xi,
\end{aligned}
$$

where the state vector $\xi \in R^{m}$ and eigenvalues of the matrix $A$ are all on the imaginary axis, which means the system is neutrally stable. Matrices $(C, A)$ are known and observable. Here, the disturbances in different subsystems are the same. Actually, different disturbances can be combined into the same form by appropriately choosing the matrix $g_{i d}\left(x_{1}, \ldots, x_{i}\right)$.

The control object is to make the closed loop system internally stable and the regulation output $\lim _{t \rightarrow \infty} e \rightarrow 0$.

For system (1), in order to continue with analysis and design, some assumptions are described as below.

Assumption 1. (1) The states $x$ are all measurable.

(2) In the field of definition, the control or virtual control gain matrix $g_{i}\left(x_{1}, x_{2}, \ldots, x_{i}\right)$ is always invertible.

Assumption 2 (see [41]). The open loop system, formulated as (1), when $D(t) \equiv 0$, is strongly forward complete. 
Assumption 3 (see [41]). The time function $\phi(t)=t-D(t)$ has these properties: when $t \geq 0, \phi(t) \leq t$ and $\phi^{\prime}(t)>0$.

Remark 4. (1) The forward completeness in Assumption 2 is used to keep the open loop system out of the systems with the finite time escape property. In order to achieve effective control, the feedback signal from the controller must reach the system in time, which is impossible for systems with finite time escape property.

(2) In Assumption 3, the property $\phi(t) \leq t$ makes the system delay function always having positive value, and the property $\phi^{\prime}(t)>0$ makes the signals sequentially injected into the system via input ports, which means that there are no feedback state values taken by the controller earlier but injected into the system later.

\section{Input Time Delay Compensation}

Consider an input delay nonlinear system, where the delay value is time varying and known as $D(t)$, described as below:

$$
\dot{x}(t)=f_{o p}(x(t), u(t-D(t))) .
$$

In the system, states $x \in R^{n}$, the control input $u \in R^{m}, t \in R_{+}$, and the differentiable function $f_{o p}$ go through the origin.

In order to adopt delay compensation, besides satisfying Assumptions 2 and 3, the open loop system without input delay needs to be asymptotically stabilizable, which is guaranteed by the following assumption in a stronger form.

Assumption 5 (see [41]). There exists a bounded feedback controller $k \in C^{1}\left(R_{+} \times R^{n}, R^{m}\right)$, which makes the nondelay (when $D(t) \equiv 0$ ) closed loop system $\dot{x}(t)=$ $f_{o p}(x(t), k(t, x(t))+\omega(t))$ ISS stable with respect to the additional input $\omega(t)$.

With system (3) satisfying Assumptions 2 and 3 and a controller $u(t)=k(t, x)$ satisfying Assumption 5, a delay compensating controller for system (3) is given as

$$
u(t)=k\left(\phi^{-1}(t), P(t)\right)
$$

where $P(t)$ makes the following equation hold:

$$
\begin{aligned}
& P(t)=\left(\phi^{-1}(t)-t\right) \int_{0}^{1} f_{o p}\left(P\left(\phi\left(t+y\left(\phi^{-1}(t)-t\right)\right)\right)\right. \\
& \left.u\left(\phi\left(t+y\left(\phi^{-1}(t)-t\right)\right)\right)\right) d y+x(t) \\
& =\int_{\phi(t)}^{t} f_{o p}(P(\sigma), u(\sigma)) \frac{d \sigma}{\phi^{\prime}\left(\phi^{-1}(\sigma)\right)} \\
& +x(t)
\end{aligned}
$$

The delay compensation control method is described as above and its performance is summarized as Lemma 6.

Lemma 6 (see [41]). With system (3), satisfying Assumptions 2 and 3, and a controller satisfying Assumption 5, a delay compensation controller ((4), (5)) makes the following inequation hold, where $\alpha$ is a KL function:

$$
\begin{aligned}
& \|x(t)\|+\sup _{\phi(t) \leq \theta \leq t}\|u(\theta)\| \\
& \quad \leq \alpha\left(\|x(0)\|+\sup _{\phi(t) \leq \theta \leq 0}\|u(\theta)\|, t\right), \quad t \geq 0 .
\end{aligned}
$$

\section{Solution of the Nonlinear Regulation Problem with Input Delay}

In this paper, for the signal to be tracked, we adopt the setting of the precise tracking: the signal to be tracked is explicitly known, whose derivations of enough high degrees can be easily obtained. The tracking and disturbance rejection problem is then transformed into the disturbance rejection problem of the tracking error system, which is a special case of the regulation problems.

Under the setting of precise tracking, the tracking error system is naturally a time-varying or parameter-varying system. For the tracking error system, in the design process the time-varying terms can always be cancelled out, which makes the Lyapunov function positive definite and its derivative negative definite uniformly with respect to time. According to the LaSalle-Yoshizawa theorem, it makes the design and proof process have no difference from a time-invariant system. So, in this paper, we treat all the systems as time-invariant systems.

To solve the output regulation problem, the controller can be divided into two parts, and we design the controller in two steps [6]. If the disturbance $d$ is measured, the steady controller can be directly formulated as a static function $c(d)$. When the disturbance $d$ is unmeasurable, after constructing an observer to asymptotically estimate the actual value of disturbance, the regulation problem can be solved by designing an observer-based controller $c(\widehat{d})$. In other words, a disturbance observer is a qualified candidate for the internal model.

4.1. Disturbance Observer. The disturbance observer for nonlinear systems is firstly presented by Chen in [53]. Here, a disturbance observer is presented with a different assumption and observer gain. Consider a nonlinear system:

$$
\begin{aligned}
\dot{x}(t) & =f(x)+g_{u}(x) u+g_{d}(x) d, \\
y & =x,
\end{aligned}
$$

where the states $x \in R^{n}$, input $u \in R^{k}$, and measured output $y \in R^{n}$. The matrices $f(x), g_{u}(x)$, and $g_{d}(x)$ are all locally Lipschitz. The gain matrix $g_{u}(x)$ is always invertible. The disturbance $d$ comes from exosystem (2). In order to design a disturbance observer, we make the following assumption.

Assumption 7. For system (7), the gain matrix $g_{d}(x)$ is known and globally invertible in the whole domain of definition. 
Then, a disturbance observer is presented as below:

$$
\begin{aligned}
\dot{z}= & \left(A-l(x) g_{d}(x) C\right) z+A p(x) \\
& \quad-l(x)\left(g_{d}(x) C p(x)+f(x)+g_{u}(x) u\right), \\
\widehat{\xi}= & z+p(x), \\
\widehat{d}= & C \widehat{\xi},
\end{aligned}
$$

where the term $l(x)$ satisfies

$$
l(x)=\frac{\partial p(x)}{\partial x}=K g_{d}^{-1}(x) .
$$

The observer designed above can estimate the disturbance $d$ exponentially as summarized in the following theorem.

Theorem 8. Considering system (7), where the disturbance d is the output of exosystem (2), when Assumption 7 is satisfied, there exist a matrix $K$ and an observer ((8), (9)) whose output is exponentially convergent to the actual disturbanced, uniformly for $x$.

Proof. Select a candidate for the Lyapunov function:

$$
V_{o}=\frac{1}{2} e^{T} e=\frac{1}{2}(\xi-\widehat{\xi})^{T}(\xi-\widehat{\xi}) .
$$

We have

$$
\begin{aligned}
\dot{V}_{o} & =e^{T}(A \xi-\dot{z}-\dot{p}(x))=e^{T}(A \xi \\
& \left.-\left(A-l(x) g_{d}(x) C\right) z-A p(x)\right)+\cdots+e^{T}(l(x) \\
& \left.\cdot\left(g_{d}(x) C p(x)+f(x)+g_{u}(x) u\right)-\frac{\partial p(x)}{\partial x} \dot{x}\right) \\
& =e^{T}(A(\xi-z-p(x))+l(x) \\
& \cdot\left(g_{d}(x) C z+g_{d}(x) C p(x)+f(x)+g_{u}(x) u\right) \\
& -l(x) \dot{x})=e^{T}(A e+l(x) \\
& \left.\cdot\left(g_{d}(x) \widehat{d}+f(x)+g_{u}(x) u-\dot{x}\right)\right)=e^{T}(A e \\
& \left.-l(x) g_{d}(x) C e\right)=e^{T}\left(A-l(x) g_{d}(x) C\right) e .
\end{aligned}
$$

According to Assumption 7 and because $(C, A)$ is observable, for any negative definite matrix $(-Q-\varepsilon I), \varepsilon>0$, there exists a matrix $K$ making $l(x)=K g_{d}^{-1}(x)$ and the following holds:

$$
\begin{aligned}
\dot{V}_{o} & =e^{T}\left(A-l(x) g_{d}(x) C\right) e=e^{T}(A-K C) e \\
& =e^{T}(-Q-\varepsilon I) e<-e^{T} Q e .
\end{aligned}
$$

With the customized positive definite matrix $Q$, we can easily choose $Q=\varepsilon_{1} I, \varepsilon_{1}>0$, which makes

$$
\dot{V}_{o}<-\varepsilon_{1} V_{o} \text {. }
$$

Then states of observer $((8),(9))$ are exponentially convergent to the exostates of the exosystem, uniformly for $x$. This also makes the estimation $\widehat{d}$ exponentially convergent to the actual disturbance $d$.
Remark 9. (1) The controller $u$ in system (7) or in observer (8) can be of any form, such as controllers with time delay or a state usually treated as a virtual controller in the backstepping method, as long as it is known.

(2) In Chen's design [53], the object system needs to be made passive by output feedback, and the observer gain matrix is designed by partial derivate. The design used in this paper needs the actual value of the invertible disturbance gain matrix and does not need any assumption about passivity.

4.2. Separation Principle. The separate principle does not hold for general nonlinear systems, and then the controllers and observers must be designed simultaneously using a single Lyapunov function for the augmented system consisting of the controlled system and the observer. Actually, the nonlinear separation principle is available, according to the Lyapunov stability theorem, when observer errors themselves are asymptotically convergent, and their transition processes do not destabilize the systems controlled by estimated-states feedback controllers. Here, we give a sufficient condition for the separation principle when dealing with a class of nonlinear system described as (7).

Theorem 10. For system (7), if the disturbance $d$ is measured and a controller $u_{\text {temp }}(x, d)$ can globally asymptotically stabilize the system; then with an additional input $v$ and an estimate $\widehat{d}$ of $d$, there exist a controller $u_{c}(x, \widehat{d})$ making system (14) ISS with respect to $(v, \tilde{d})$; furthermore if the estimation $\widehat{d}$ is exponentially convergent to the disturbance $d$ and, in system (14), the inputto-states gain $\lambda_{2}^{-1}$ with respect to $\tilde{d}$ satisfies $\lambda_{2}^{-1} I<C^{T} C$, then $u_{c}(x, \widehat{d})$ asymptotically stabilize the augmented system of (7) and the disturbance estimation error system, which means the separation principle is available.

$$
\begin{aligned}
\dot{x}(t) & =f(x)+g_{u}(x)\left(u_{c}+v\right)+g_{d}(x) \hat{d}+g_{d}(x) \tilde{d}, \\
y & =x .
\end{aligned}
$$

Proof. When $d$ is known there exists the controller $u_{\text {temp }}(x, d)$ globally asymptotically stabilizing system (7) and then there is a Lyapunov function $V(x)$, which makes

$$
\begin{aligned}
& \underline{\alpha}(\|x\|) \leq V(x) \leq \bar{\alpha}(\|x\|), \\
& \frac{\partial V(x)}{\partial x}\left(f(x)+g_{u}(x) u_{\mathrm{temp}}(x, d)+g_{d}(x) d\right) \\
& \quad \leq-\beta(\|x\|),
\end{aligned}
$$

where $\underline{\alpha}, \bar{\alpha}$, and $\beta$ belong to the $K$-class functions.

Invoke $u_{c}(x, \widehat{d})=u_{\text {temp }}(x, \widehat{d})+u_{a d}(x, \widehat{d})$ into system (14), and then we have

$$
\begin{aligned}
\dot{x}(t)= & f(x)+g_{u}(x) u_{\text {temp }}(x, \widehat{d})+g_{d}(x) \hat{d}+\cdots \\
& +g_{u}(x) u_{a d}(x, \widehat{d})+g_{u}(x) v+g_{d}(x) \tilde{d} .
\end{aligned}
$$


Invoking the controller

$$
\begin{aligned}
& u_{a d}(x, \widehat{d}) \\
& =-\left(c_{1} g_{u}^{T}(x)+c_{2} g_{u}^{-1}(x) g_{d}(x) g_{d}^{T}(x)\right)\left(\frac{\partial V(x)}{\partial x}\right)^{T} .
\end{aligned}
$$

$u_{\text {temp }}$ in equation (16) is different from the one in equation (15), and we can put the difference term $u_{\text {temp }}(x, d)-$ $u_{\text {temp }}(x, \hat{d})$ into the additional input $v$. Then $u_{\text {temp }}$ in (18) can be treated the same as the one in (15). The derivative of the Lyapunov function candidate $V(x)$ along the system (16) satisfies

$$
\begin{aligned}
& \dot{V}(x)=\frac{\partial V(x)}{\partial x}\left(f(x)+g_{u}(x) u_{\mathrm{temp}}+g_{d}(x) \hat{d}\right. \\
& \left.+g_{u}(x) u_{a d}+g_{u}(x) v+g_{d}(x) \tilde{d}\right) \leq-\beta(\|x\|) \\
& +\frac{\partial V(x)}{\partial x}\left(-\left(c_{1} g_{u}^{2}(x)+c_{2} g_{d}^{2}(x)\right)\left(\frac{\partial V(x)}{\partial x}\right)^{T}\right. \\
& \left.+g_{u}(x) v+g_{d}(x) \tilde{d}\right) \leq-\beta(\|x\|) \\
& +c_{1}\left\|\frac{\partial V(x)}{\partial x} g_{u}(x)\right\|^{2}-c_{2}\left\|\frac{\partial V(x)}{\partial x} g_{d}(x)\right\|^{2} \\
& +\lambda_{1}\left\|\frac{\partial V(x)}{\partial x} g_{u}(x)\right\|^{2}+\cdots+\lambda_{1}^{-1}\|v\|^{2} \\
& +\lambda_{2}\left\|\frac{\partial V(x)}{\partial x} g_{d}(x)\right\|^{2}+\lambda_{2}^{-1}\|\tilde{d}\|^{2} \leq-\beta(\|x\|)-\left(c_{1}\right. \\
& +\cdots+\lambda_{1}^{-1}\|v\|^{2}+\lambda_{2}^{-1}\|\tilde{d}\|^{2}, \\
& \left.+\lambda_{1}\right)\left\|\frac{\partial V(x)}{\partial x} g_{u}(x)\right\|^{2}-\left(c_{2}-\lambda_{2}\right)\left\|\frac{\partial V(x)}{\partial x} g_{d}(x)\right\|^{2}
\end{aligned}
$$

where the scalars $c_{1}$ and $c_{2}$ are designing parameters. Given any $\lambda_{1}>0$ and $\lambda_{2}>0$, select $c_{1}$ and $c_{2}$ making inequations $\left(c_{1}-\lambda_{1}\right)>0$ and $\left(c_{2}-\lambda_{2}\right)>0$ hold, and then we have

$$
\begin{aligned}
\dot{V}(x) \leq & -\beta(\|x\|)-\left(c_{1}-\lambda_{1}\right)\left\|\frac{\partial V(x)}{\partial x} g_{u}(x)\right\|^{2} \\
& -\left(c_{2}-\lambda_{2}\right)\left\|\frac{\partial V(x)}{\partial x} g_{d}(x)\right\|^{2}+\cdots+\lambda_{1}^{-1}\|v\|^{2} \\
& +\lambda_{2}^{-1}\|\tilde{d}\|^{2} \\
< & -\beta(\|x\|)+\lambda_{1}^{-1}\|v\|^{2}+\lambda_{2}^{-1}\|\tilde{d}\|^{2} .
\end{aligned}
$$

It shows system $(14)$ is ISS with respect to $(v, \widetilde{d})$.

When the observer error is exponentially convergent uniformly, according to the proof of Theorem 8, the Lyapunov function $V_{o}=(1 / 2) e^{T} e$, and its derivative $\dot{V}_{o}<-e^{T} Q e$. Because $(C, A)$ is observable, so the matrix $C^{T} C$ is positive definite. Then, take a Lyapunov function candidate

$$
V_{d}=\frac{1}{2} e^{T} C^{T} C e=\frac{1}{2} \widetilde{d}^{T} \widetilde{d}
$$

We have

$$
\dot{V}_{d}=e^{T} C^{T} C \dot{e}<-e^{T} C^{T} C Q e .
$$

With $Q$ selectable, choose $Q=C^{T} C>\mu I$, where $\mu$ is a positive constant. Then we have

$$
\dot{V}_{d}<-e^{T} C^{T} C C^{T} C e=-\widetilde{d}^{T} C C^{T} \widetilde{d}^{T}<-\mu\|\widetilde{d}\|^{2} .
$$

The original system to be controlled and the dynamic of the disturbance observer error form the augmented system with augmented states $(x, \tilde{d})$. Take a candidate for Lyapunov function of the augmented system as $V_{(x, \tilde{d})}=V_{d}+V$, whose derivative shows

$$
\begin{aligned}
\dot{V}_{(x, \tilde{d})} & =\dot{V}_{d}+\dot{V} \\
& <-\beta(\|x\|)+\lambda_{1}^{-1}\|v\|^{2}+\lambda_{2}^{-1}\|\widetilde{d}\|^{2}-\mu\|\widetilde{d}\|^{2} .
\end{aligned}
$$

If $v=0$, the value of $\lambda_{2}^{-1}$ can be set as $\lambda_{2}^{-1}=\mu-\mu_{0}, \mu_{0}>0$, and then

$$
\dot{V}_{(x, \tilde{d})}<-\beta(\|x\|)-\mu_{0}\|\widetilde{d}\|^{2} .
$$

So, when the observer states are exponentially convergent to the exostates of exosystem uniformly for $x$ and the input-to-states gain $\lambda_{2}^{-1}$ with respect to $\widetilde{d}$ satisfies $\lambda_{2}^{-1} I<$ $C^{T} C$, controller (25) asymptotically stabilizes the augmented system of (7) and the dynamic of the disturbance observer error. The separation principle is available.

$$
\begin{aligned}
u_{c}(x, \widehat{d})= & u_{\text {temp }}(x, \widehat{d}) \\
& -\left(c_{1} g_{u}^{T}(x)+c_{2} g_{u}^{-1}(x) g_{d}(x) g_{d}^{T}(x)\right) \\
& \cdot\left(\frac{\partial V(x)}{\partial x}\right)^{T} .
\end{aligned}
$$

Remark 11 . The IS-gain $\lambda_{2}^{-1}$ can be easily designed to satisfy the condition $\lambda_{2}^{-1} I<C^{T} C$. This condition is nontrivial and can not be ignored.

4.3. Controller Design and Stability Analysis. In order to solve the regulation problem described in Section 2, we firstly solve the regulation problem without input delay, as $D(t) \equiv 0$, and design a controller based on the backstepping method and the disturbance observer; then, using the ISS property of the designed controller we construct the final delay compensation controller based on Lemma 6.

For system (1), reference signal $y_{r}$ and its derivation $y_{r}^{(i)}$ are all known, and a tracking controller with ISS properties is designed as follows.

In the first step, define the output tracking error as $z_{1}=$ $x_{1}-y_{r}$, and its dynamic is described as

$$
\dot{z}_{1}=\dot{x}_{1}-\dot{y}_{r}=f_{1}\left(x_{1}\right)+g_{1}\left(x_{1}\right) x_{2}+g_{1 d}\left(x_{1}\right) d-\dot{y}_{r} .
$$


Define a virtual controller $a_{1}$ :

$$
\begin{aligned}
\dot{z}_{1}= & f_{1}\left(x_{1}\right)+g_{1}\left(x_{1}\right)\left(a_{1}+\left(x_{2}-a_{1}\right)\right)+g_{1 d}\left(x_{1}\right) d \\
& -\dot{y}_{r} .
\end{aligned}
$$

Then, define a tracking error with respect to the virtual controller as $z_{2}=x_{2}-a_{1}$, and we have

$$
\dot{z}_{1}=f_{1}\left(x_{1}\right)+g_{1}\left(x_{1}\right)\left(a_{1}+z_{2}\right)+g_{1 d}\left(x_{1}\right) d-\dot{y}_{r} .
$$

Using an exponentially convergent disturbance observer and according to Theorem 10, there exists a virtual controller $a_{1}\left(y_{r}, \dot{y}_{r}, x_{1}, \widehat{d}\right)$ described as below:

$$
\begin{aligned}
a_{1} & =u_{\text {temp } 1} \\
& -\left(c_{a_{1}} g_{1}^{T}\left(x_{1}\right)+c c_{a_{1}} g_{1}^{-1}\left(x_{1}\right) g_{1 d}\left(x_{1}\right) g_{1 d}^{T}\left(x_{1}\right)\right) \\
& \cdot\left(\frac{\partial V_{1}\left(z_{1}\right)}{\partial z_{1}}\right)^{T}
\end{aligned}
$$

which makes $z_{1}$ subsystem ISS with respect to $z_{2}$ and $\tilde{d}$, where $\widehat{d}$ and $\widetilde{d}=d-\widehat{d}$ are the estimate value of $d$ and its estimate error, respectively. In (29), $V_{1}\left(z_{1}\right)$ is a CLF (Control Lyapunov Function) for $z_{1}$ subsystem and $u_{\text {temp } 1}\left(y_{r}, \dot{y}_{r}, x_{1}, \widehat{d}\right)$ is an arbitrary asymptotical stabilizer. They can be chosen as

$$
\begin{aligned}
& V_{1}\left(z_{1}\right)=\frac{1}{2} z_{1}^{T} z_{1}, \\
& u_{\text {temp } 1}=g_{1}^{-1}\left(x_{1}\right)\left(\dot{y}_{r}-f_{1}\left(x_{1}\right)-k_{1} z_{1}-g_{1 d}\left(x_{1}\right) \widehat{d}\right) .
\end{aligned}
$$

In the second step, the dynamic of the tracking error with respect to the first virtual controller is

$$
\begin{aligned}
\dot{z}_{2}= & \dot{x}_{2}-\frac{\partial a_{1}}{\partial x_{1}}\left(f_{1}\left(x_{1}\right)+g_{1}\left(x_{1}\right) x_{2}+g_{1 d}\left(x_{1}\right) d\right) \\
& -\frac{\partial a_{1}}{\partial y_{r}} \dot{y}_{r}-\frac{\partial a_{1}}{\partial \dot{y}_{r}} y_{r}^{(2)}-\frac{\partial a_{1}}{\partial \hat{d}} \\
= & f_{2}\left(\bar{x}_{2}\right)+g_{2}\left(\bar{x}_{2}\right) x_{3}+g_{2 d}\left(\bar{x}_{2}\right) d \\
& -\frac{\partial a_{1}}{\partial x_{1}}\left(f_{1}\left(x_{1}\right)+g_{1}\left(x_{1}\right) x_{2}+g_{1 d}\left(x_{1}\right) d\right) \\
& -\frac{\partial a_{1}}{\partial y_{r}} \dot{y}_{r}-\frac{\partial a_{1}}{\partial \dot{y}_{r}} y_{r}^{(2)}-\frac{\partial a_{1}}{\partial \hat{d}} \dot{\vec{d}}
\end{aligned}
$$

Defining a tracking error as $z_{3}=x_{3}-a_{2}$, (31) becomes as

$$
\begin{aligned}
\dot{z}_{2}= & f_{2}\left(\bar{x}_{2}\right)+g_{2}\left(\bar{x}_{2}\right)\left(a_{2}+z_{3}\right)+g_{2 d}\left(\bar{x}_{2}\right) d \\
& -\frac{\partial a_{1}}{\partial x_{1}}\left(f_{1}\left(x_{1}\right)+g_{1}\left(x_{1}\right) x_{2}+g_{1 d}\left(x_{1}\right) d\right) \\
& -\frac{\partial a_{1}}{\partial y_{r}} \dot{y}_{r}-\frac{\partial a_{1}}{\partial \dot{y}_{r}} y_{r}^{(2)}-\frac{\partial a_{1}}{\partial \hat{d}} \dot{\vec{d}} .
\end{aligned}
$$

The same as in the first step, there exists a virtual controller $a_{2}\left(\bar{y}_{r}^{(2)}, \bar{x}_{2}, \overline{\widehat{d}}^{(1)}\right)$ described as below:

$$
\begin{aligned}
a_{2} & =u_{\text {temp } 2}-\left(c_{a_{2}} g_{2}^{T}\left(\bar{x}_{2}\right)\right. \\
& \left.+c c_{a_{2}} g_{2}^{-1}\left(\bar{x}_{2}\right)\left(g_{2 d}\left(\bar{x}_{2}\right)-\frac{\partial a_{1}}{\partial x_{1}} g_{1 d}\left(x_{1}\right)\right)^{2}\right) \\
& \cdot\left(\frac{\partial V_{2}\left(z_{2}\right)}{\partial z_{2}}\right)^{T}
\end{aligned}
$$

which makes $z_{2}$ subsystem ISS with respect to $z_{3}$ and $\tilde{d}$. In (33), $V_{2}\left(z_{2}\right)$ is a CLF for $z_{2}$ subsystem and $u_{\text {temp2 }}\left(\bar{y}_{r}^{(2)}, \bar{x}_{2}, \overline{\widehat{d}}^{(1)}\right)$ is an arbitrary asymptotical stabilizer. They can be chosen as

$$
\begin{aligned}
& V_{2}\left(z_{2}\right)=\frac{1}{2} z_{2}^{T} z_{2} \\
& u_{\mathrm{temp} 2}=g_{2}^{-1}\left(\bar{x}_{2}\right) \\
& \cdot\left(\frac{\partial a_{1}}{\partial x_{1}}\left(f_{1}\left(x_{1}\right)+g_{1}\left(x_{1}\right) x_{2}+g_{1 d}\left(x_{1}\right) \hat{d}\right)\right. \\
& +\frac{\partial a_{1}}{\partial y_{r}} \dot{y}_{r}+\cdots+\frac{\partial a_{1}}{\partial \dot{y}_{r}} y_{r}^{(2)}+\frac{\partial a_{1}}{\partial \widehat{d}} \dot{\hat{d}}-f_{2}\left(\bar{x}_{2}\right)-k_{2} z_{2} \\
& \left.-g_{2 d}\left(\bar{x}_{2}\right) \hat{d}\right)
\end{aligned}
$$

The same as in the standard backstepping design process, the virtual controller's tracking error cross-term in the upper subsystem can be cancelled out with a state feedback term in the lower subsystem. So, there is no need to make every $z_{i}$ subsystem ISS with respect to the virtual controller's tracking error $z_{i+1}$. To achieve the counteraction for the crossterm, modify $u_{\text {temp2 }}\left(\bar{y}_{r}^{(2)}, \bar{x}_{2}, \overline{\widehat{d}}^{(1)}\right)$ in the virtual controller $a_{2}\left(\bar{y}_{r}^{(2)}, \bar{x}_{2}, \overline{\widehat{d}}^{(1)}\right)$ as

$$
\begin{aligned}
& u_{\text {temp } 2}=g_{2}^{-1}\left(\bar{x}_{2}\right)\left(-g_{1}^{T}\left(x_{1}\right) z_{1}\right. \\
& +\frac{\partial a_{1}}{\partial x_{1}}\left(f_{1}\left(x_{1}\right)+g_{1}\left(x_{1}\right) x_{2}+g_{1 d}\left(x_{1}\right) \widehat{d}\right)+\frac{\partial a_{1}}{\partial y_{r}} \dot{y}_{r} \\
& +\cdots+\frac{\partial a_{1}}{\partial \dot{y}_{r}} y_{r}^{(2)}+\frac{\partial a_{1}}{\partial \hat{d}} \dot{\hat{d}}-f_{2}\left(\bar{x}_{2}\right)-k_{2} z_{2} \\
& \left.-g_{2 d}\left(\bar{x}_{2}\right) \hat{d}\right)
\end{aligned}
$$

and set $c_{a_{1}}=0, c_{a_{2}}=0, c c_{a_{1}}>0$, and $c c_{a_{2}}>0$. Hereafter, it is handled the same. 
In the $i$ th step, $i \geq 3$, the dynamic of the tracking error $z_{i}=x_{i}-a_{i-1}$ is

$\dot{z}_{i}$

$$
\begin{aligned}
= & f_{i}\left(\bar{x}_{i}\right)+g_{i}\left(\bar{x}_{i}\right)\left(a_{i}+z_{i+1}\right)+g_{i d}\left(\bar{x}_{i}\right) d \\
& -\sum_{k=0}^{i-1} \frac{\partial a_{i-1}}{\partial y_{r}^{(k)}} y_{r}^{(k+1)}-\cdots-\sum_{k=0}^{i-2} \frac{\partial a_{i-1}}{\partial \hat{d}^{(k)}} \hat{d}^{(k+1)} \\
& -\sum_{k=1}^{i-1} \frac{\partial a_{i-1}}{\partial x_{k}}\left(f_{k}\left(\bar{x}_{k}\right)+g_{k}\left(\bar{x}_{k}\right) x_{k+1}+g_{k d}\left(\bar{x}_{k}\right) d\right),
\end{aligned}
$$

where $\bar{x}_{i}=\left(x_{1}, \ldots, x_{i}\right), \bar{y}_{r}^{(i)}=\left(y_{r}, \dot{y}_{r}, \ldots, y_{r}^{(i)}\right), \overline{\hat{d}}^{(i)}=(\widehat{d}, \dot{\hat{d}}$, $\left.\ldots, \widehat{d}^{(i)}\right)$, and $z_{i+1}=x_{i+1}-a_{i}$. According to Theorem 10, there exists a virtual controller $a_{i}\left(\bar{y}_{r}^{(i)}, \bar{x}_{i}, \overline{\widehat{d}}^{(i-1)}\right)$ described as below:

$$
\begin{aligned}
a_{i}= & u_{\text {tempi }}-\left(c_{a_{i}} g_{i}^{T}\left(\bar{x}_{i}\right)\right. \\
& \left.+c c_{a_{i}} g_{i}^{-1}\left(\bar{x}_{i}\right)\left(g_{i d}\left(\bar{x}_{i}\right)-\sum_{k=1}^{i-1} \frac{\partial a_{i-1}}{\partial x_{k}} g_{k d}\left(\bar{x}_{k}\right)\right)^{2}\right) \\
& \cdot\left(\frac{\partial V_{i}\left(z_{i}\right)}{\partial z_{i}}\right)^{T}
\end{aligned}
$$

which makes $z_{i}$ subsystem ISS to $z_{i+1}$ and $\tilde{d}$. In (37), $V_{i}\left(z_{i}\right)$ is a CLF for $z_{i}$ subsystem and $u_{\text {tempi }}\left(\bar{y}_{r}^{(i)}, \bar{x}_{i}, \overline{\widehat{d}}^{(i-1)}\right)$ is an arbitrary asymptotical stabilizer. Similarly, we can choose

$$
\begin{aligned}
& V_{i}\left(z_{i}\right)=\frac{1}{2} z_{i}^{T} z_{i} \\
& u_{\text {tempi }}=g_{i}^{-1}\left(\bar{x}_{i}\right)\left(-g_{i-1}^{T}\left(\bar{x}_{i-1}\right) z_{i-1}-f_{i}\left(\bar{x}_{i}\right)-k_{i} z_{i}\right. \\
& \quad-g_{i d}\left(\bar{x}_{i}\right) \hat{d}+\sum_{k=0}^{i-1} \frac{\partial a_{i-1}}{\partial y_{r}^{(k)}} y_{r}^{(k+1)}+\cdots+\sum_{k=0}^{i-2} \frac{\partial a_{i-1}}{\partial \widehat{d}^{(k)}} \widehat{d}^{(k+1)} \\
& \left.\quad+\sum_{k=1}^{i-1} \frac{\partial a_{i-1}}{\partial x_{k}}\left(f_{k}\left(\bar{x}_{k}\right)+g_{k}\left(\bar{x}_{k}\right) x_{k+1}+g_{k d}\left(\bar{x}_{k}\right) \widehat{d}\right)\right) .
\end{aligned}
$$

Set $c_{a_{i}}=0$ and $c c_{a_{i}}>0$.

In the $n$th step, the tracking error $z_{n}=x_{n}-a_{n-1}$, and its dynamic is

$$
\begin{aligned}
\dot{z}_{n}= & \dot{x}_{n}-\dot{a}_{n-1} \\
= & f_{n}\left(\bar{x}_{n}\right)+g_{n}\left(\bar{x}_{n}\right) u+g_{n d}\left(\bar{x}_{n}\right) d-\sum_{k=0}^{n-1} \frac{\partial a_{n-1}}{\partial y_{r}^{(k)}} y_{r}^{(k+1)} \\
& -\cdots-\sum_{k=0}^{n-2} \frac{\partial a_{n-1}}{\partial \widehat{d}^{(k)}} \widehat{d}^{(k+1)} \\
& -\sum_{k=1}^{n-1} \frac{\partial a_{n-1}}{\partial x_{k}}\left(f_{k}\left(\bar{x}_{k}\right)+g_{k}\left(\bar{x}_{k}\right) x_{k+1}+g_{k d}\left(\bar{x}_{k}\right) d\right) .
\end{aligned}
$$

According to Theorem 10, there exists a controller $u\left(\bar{y}_{r}^{(n)}, \bar{x}_{n}\right.$, $\overline{\widehat{d}}^{(n-1)}$ ) described as below:

$$
\begin{aligned}
u= & u_{\text {tempn }}-\left(c_{a_{n}} g_{n}^{T}\left(\bar{x}_{n}\right)\right. \\
& \left.+c c_{a_{n}} g_{n}^{-1}\left(\bar{x}_{n}\right)\left(g_{n d}\left(\bar{x}_{n}\right)-\sum_{k=1}^{n-1} \frac{\partial a_{n-1}}{\partial x_{k}} g_{k d}\left(\bar{x}_{k}\right)\right)^{2}\right) \\
& \cdot\left(\frac{\partial V_{n}\left(z_{n}\right)}{\partial z_{n}}\right)^{T}
\end{aligned}
$$

which makes $z_{n}$ subsystem ISS with respect to the additional input $v$ and $\widetilde{d} . V_{n}\left(z_{n}\right)$ and $u_{\text {tempn }}\left(\bar{y}_{r}^{(n)}, \bar{x}_{n}, \overline{\hat{d}}^{(n-1)}\right)$ are chosen as

$$
\begin{aligned}
& V_{n}\left(z_{n}\right)=\frac{1}{2} z_{n}^{T} z_{n}, \\
& u_{\text {tempn }}=g_{n}^{-1}\left(\bar{x}_{n}\right)\left(-g_{n-1}^{T}\left(\bar{x}_{n-1}\right) z_{n-1}-f_{n}\left(\bar{x}_{n}\right)\right. \\
& \quad-k_{n} z_{n}-g_{n d}\left(\bar{x}_{n}\right) \widehat{d}+\sum_{k=0}^{n-1} \frac{\partial a_{i-1}}{\partial y_{r}^{(k)}} y_{r}^{(k+1)}+\cdots \\
& \quad+\sum_{k=0}^{n-2} \frac{\partial a_{i-1}}{\partial \widehat{d}^{(k)}} \hat{d}^{(k+1)}+\sum_{k=1}^{n-1} \frac{\partial a_{i-1}}{\partial x_{k}}\left(f_{k}\left(\bar{x}_{k}\right)\right. \\
& \left.\left.\quad+g_{k}\left(\bar{x}_{k}\right) x_{k+1}+g_{k d}\left(\bar{x}_{k}\right) \hat{d}\right)\right) .
\end{aligned}
$$

Different from the previous steps, here set $c_{a_{n}}>0$ and $c c_{a_{n}}>0$.

The closed loop system obtained from the above process has a property described as Theorem 12 .

Theorem 12. Considering system (1), the controller $u\left(\bar{y}_{r}^{(n)}\right.$, $\bar{x}_{n}, \overline{\widehat{d}}^{(n-1)}$ ) as in formula (40) and (42), and virtual controllers $a_{i}\left(\bar{y}_{r}^{(i)}, \bar{x}_{i}, \overline{\hat{d}}^{(i-1)}\right)$ given above, the closed loop system (43) is made ISS with respect to the additional input $v$ and $\tilde{d}$.

$$
\begin{aligned}
& \dot{z}_{1}=f_{1}\left(x_{1}\right)+g_{1}\left(x_{1}\right)\left(a_{1}+z_{2}\right)+g_{1 d}\left(x_{1}\right)(\hat{d}+\tilde{d}) \\
& -\dot{y}_{r}, \\
& \dot{z}_{i}=f_{i}\left(\bar{x}_{i}\right)+g_{i}\left(\bar{x}_{i}\right)\left(a_{i}+z_{i+1}\right)+g_{i d}\left(\bar{x}_{i}\right)(\hat{d}+\widetilde{d}) \\
& -\dot{a}_{i-1}, \\
& \dot{z}_{n}=f_{n}\left(\bar{x}_{n}\right)+g_{n}\left(\bar{x}_{n}\right)(u+v)+g_{n d}\left(\bar{x}_{n}\right)(\widehat{d}+\widetilde{d}) \\
& -\dot{a}_{n-1} \text {, } \\
& y_{c}=z_{1} .
\end{aligned}
$$


Proof. Take a Lyapunov function candidate as

$$
V\left(\bar{z}_{n}\right)=\sum_{k=1}^{n} V_{k}\left(z_{k}\right)=\frac{1}{2} \bar{z}_{n}^{T} \bar{z}_{n},
$$

where $\bar{z}_{n}=\left(z_{1}, \ldots, z_{n}\right)^{T}$ :

$$
\begin{aligned}
& \dot{V}\left(\bar{z}_{n}\right)=\sum_{k=1}^{n} z_{k}^{T} \dot{z}_{k}=z_{1}^{T}\left(f_{1}\left(x_{1}\right)+g_{1}\left(x_{1}\right)\left(a_{1}+z_{2}\right)\right. \\
& \left.\quad+g_{1 d}\left(x_{1}\right) d-\dot{y}_{r}\right)+\cdots+\sum_{k=2}^{n-1} z_{k}^{T}\left(f_{k}\left(\bar{x}_{k}\right)\right. \\
& \left.\quad+g_{k}\left(\bar{x}_{k}\right)\left(a_{k}+z_{k+1}\right)+g_{k d}\left(\bar{x}_{k}\right)(\widehat{d}+\tilde{d})-\dot{a}_{k-1}\right) \\
& +\cdots+z_{n}^{T}\left(f_{n}\left(\bar{x}_{n}\right)+g_{n}\left(\bar{x}_{n}\right)(u+v)+g_{n d}\left(\bar{x}_{n}\right)(\widehat{d}\right. \\
& \left.\quad+\tilde{d})-\dot{a}_{n-1}\right) .
\end{aligned}
$$

Invoking the controller $u\left(\bar{y}_{r}^{(n)}, \bar{x}_{n}, \overline{\widehat{d}}^{(n-1)}\right)$ as in formula (40) and (42), and virtual controllers $a_{i}\left(\bar{y}_{r}^{(i)}, \bar{x}_{i}, \overline{\widehat{d}}^{(i-1)}\right)$ given in the above process, we have

$$
\begin{aligned}
& \dot{V}\left(\bar{z}_{n}\right)=z_{1}^{T}\left(-k_{1} z_{1}+g_{1}\left(x_{1}\right) z_{2}+g_{1 d}\left(x_{1}\right) \tilde{d}\right. \\
& \left.-c c_{a_{1}} g_{1 d}^{2}\left(x_{1}\right)\left(\frac{\partial V_{1}\left(z_{1}\right)}{\partial z_{1}}\right)^{T}\right)+\cdots \\
& +\sum_{k=2}^{n-1} z_{k}^{T}\left(-k_{k} z_{k}-g_{k-1}^{T}\left(\bar{x}_{k-1}\right) z_{k-1}+g_{k}\left(\bar{x}_{k}\right) z_{k+1}\right. \\
& +g_{k d}\left(\bar{x}_{k}\right) \tilde{d}-\sum_{p=1}^{k-1} \frac{\partial a_{k-1}}{\partial x_{p}} g_{p d}\left(\bar{x}_{p}\right) \tilde{d}-\cdots \\
& +c c_{a_{k}}\left(g_{k d}\left(\bar{x}_{k}\right)-\sum_{p=1}^{k-1} \frac{\partial a_{k-1}}{\partial x_{p}} g_{p d}\left(\bar{x}_{p}\right)\right)^{2} \\
& \left.+\left(\frac{\partial V_{n}\left(z_{n}\right)}{\partial z_{n}}\right)^{T}\right) \cdot \\
& \left.+g_{a_{n}}\left(g_{n d}\left(\bar{x}_{n}\right)-\sum_{p=1}^{n-1} \frac{\partial a_{n-1}}{\partial x_{p}} g_{p d}\left(\bar{x}_{p}\right)\right)^{2}\right)+\cdots+z_{n}^{T}\left(-k_{n} z_{n}-g_{n-1}^{T}\left(\bar{x}_{n-1}\right)\right. \\
& +z_{n-1}+g_{n d}\left(\bar{x}_{n}\right) \tilde{d}-\sum_{p=1}^{n-1} \frac{\partial a_{k-1}}{\partial x_{p}} g_{p d}\left(\bar{x}_{p}\right) \tilde{d} \\
& +\left(c_{a_{n}} g_{n}^{2}\left(\bar{x}_{n}\right)\right. \\
& + \\
& +\left(z_{n}\right)
\end{aligned}
$$

Using Young's inequation, we have

$$
\begin{aligned}
& \dot{V}\left(\bar{z}_{n}\right)=-\sum_{k=1}^{n} k_{k} z_{k}^{T} z_{k}+z_{1}^{T} g_{1 d}\left(x_{1}\right) \tilde{d} \\
& -c c_{a_{1}}\left\|z_{1}^{T} g_{1 d}\left(x_{1}\right)\right\|^{2}+\cdots \\
& +\sum_{k=2}^{n}\left(z_{k}^{T}\left(g_{k d}\left(\bar{x}_{k}\right)-\sum_{p=1}^{k-1} \frac{\partial a_{k-1}}{\partial x_{p}} g_{p d}\left(\bar{x}_{p}\right)\right) \tilde{d}\right. \\
& \left.-c c_{a_{k}}\left\|z_{k}^{T}\left(g_{k d}\left(\bar{x}_{k}\right)-\sum_{p=1}^{k-1} \frac{\partial a_{k-1}}{\partial x_{p}} g_{p d}\left(\bar{x}_{p}\right)\right)\right\|^{2}\right) \\
& +z_{n}^{T}\left(-c_{a_{n}} g_{n}^{2}\left(\bar{x}_{n}\right) z_{n}+g_{n}\left(\bar{x}_{n}\right) v\right) \leq-\sum_{k=1}^{n} k_{k}\left\|z_{k}\right\|^{2} \\
& -\left(c c_{a_{1}}-\lambda_{1}\right)\left\|z_{1}^{T} g_{1 d}\left(x_{1}\right)\right\|^{2}+\sum_{k=1}^{n} \frac{1}{\lambda_{k}}\|\tilde{d}\|^{2}-\cdots \\
& -\sum_{k=2}^{n}\left(c c_{a_{k}}-\lambda_{k}\right) \| z_{k}^{T}\left(g_{k d}\left(\bar{x}_{k}\right)\right. \\
& \left.-\sum_{p=1}^{k-1} \frac{\partial a_{k-1}}{\partial x_{p}} g_{p d}\left(\bar{x}_{p}\right)\right)\left\|^{2}-\left(c_{a_{n}}-\gamma\right)\right\| z_{n}^{T} g_{n}\left(\bar{x}_{n}\right) \|^{2} \\
& +\frac{1}{\gamma}\|v\|^{2} .
\end{aligned}
$$

From (47), it is shown that, for any $\lambda_{i}>0, \gamma>0, k_{i}>0$, $c c_{a_{i}} \geq \lambda_{i}$, and $c_{a_{n}} \geq \gamma$, the states $\bar{z}_{n}$ of system (43) are ISS with respect to the additional input $v$ and the disturbance observer error $\widetilde{d}$.

Then, based on Assumption 5 and Lemma 6, an input delay compensation controller is given as below:

$$
u\left(\bar{y}_{r}^{(n)}, P(t), \overline{\bar{d}}^{(n-1)}\right),
$$

where the term $P(t)$ is the solution of

$$
\begin{aligned}
& P(t)=\left(\phi^{-1}(t)-t\right) \\
& \cdot \int_{0}^{1} f_{(1)}\left(P\left(\phi\left(t+y\left(\phi^{-1}(t)-t\right)\right)\right),\right. \\
& \left.u\left(\phi\left(t+y\left(\phi^{-1}(t)-t\right)\right)\right)\right) d y+\bar{x}_{n}(t) \\
& =\int_{\phi(t)}^{t} f_{(1)}(P(\sigma), u(\sigma)) \frac{d \sigma}{\phi^{\prime}\left(\phi^{-1}(\sigma)\right)} \\
& +\bar{x}_{n}(t) .
\end{aligned}
$$

The function $f_{(1)}(\cdot)$ represents the right side of system (1). The estimation $\widehat{d}$ is given by the disturbance observer presented in (8) and (9).

Based on Lemma 6 and Theorems 8, 10, and 12, we can easily get the following corollary. 
Corollary 13. For system (1), satisfying Assumptions 2 and 3, the delay compensation controller (40), (42), (48), and (49) makes the following inequation hold, where $\alpha$ is a KL class function:

$$
\begin{aligned}
& \|x(t)\|+\sup _{\phi(t) \leq \theta \leq t}\|u(\theta)\| \\
& \quad \leq \alpha\left(\|x(0)\|+\sup _{\phi(t) \leq \theta \leq 0}\|u(\theta)\|, t\right), \quad t \geq 0 .
\end{aligned}
$$

Proof. In Theorem 12, system (1) is shown to be ISS with respect to the additional input $v$ and the disturbance observer error $\tilde{d}$. In Theorem 8 , the observer error is shown to be exponentially convergent and we can easily choose $\varepsilon>0$ to make the augmented system (1) and (2) ISS with respect to $v$. Then, according to Lemma 6 , the delay compensation controller (40), (42), (48), and (49) makes inequation (50) hold.

\section{AHV Attitude Control}

5.1. AHV Control System Description. According to the dynamic equation and kinematics equation of attitude motion of an AHV, when time delay caused by signal transmission and actuator dynamics is abstracted as an input delay, the control problem of AHV attitude angles with respect to the air-path axis system can be described as a control design problem of a system in the states strict feedback form, which is presented as below:

$$
\begin{aligned}
& \dot{\Omega}=f_{s}+g_{s} \omega+d, \\
& \dot{\omega}=f_{f}+g_{f} g_{f \delta} \delta(t-D(t))+d, \\
& y=\Omega .
\end{aligned}
$$

The nonlinear function vectors, $f_{s}$ and $f_{f}$, and the virtual and actual control gain matrices, $g_{s}$ and $g_{f} g_{\delta}$, are all known. The disturbance $d$ is given by the exosystem model (2). The aerodynamic model is given by the NASA Langley Research Center in [54]. The model of time delay $D(t)$ is assumed to be known. Here, for simplicity, the disturbance gain matrices are set as the identity matrix. The control object is to design a controller making the system output track the reference signal $y_{d}(t)$ and keep all the states bounded. For research convenience and considering actual situations, some assumptions are given as below.

Assumption 14. (1) All the states, $\Omega$ and $\omega$, which means the angle of attack, the angle of sideslip, the angle around the roll speed, and elements of the angular velocity in the body frame, are all measured. The reference output signal $y_{d}(t)$ is exactly known, which is continue enough and bounded, and its derivatives with enough degrees are also known, continuous, and bounded.

(2) The virtual control gain matrix $g_{s}$ and the actual control gain matrix $g_{f} g_{f \delta}$ are all invertible in the whole domain.

(3) The AHV attitude system (51) fulfills Assumption 2 when the delay is relatively small.
The problem is solved using the method presented above. Based on the separation principle proved, disturbance observers can be designed independently as

$$
\begin{aligned}
& \dot{z}_{1}=\left(A_{1}-K_{1} C_{1}\right) z_{1}+A_{1} K_{1} \Omega \\
&-K_{1}\left(C_{1} K_{1} \Omega+f_{s}+g_{s} \omega\right), \\
& \hat{\xi}_{1}= z_{1}+K_{1} \Omega, \\
& \widehat{d}_{1}=C_{1} \widehat{\xi}_{1}, \\
& \dot{z}_{2}=\left(A_{2}-K_{2} C_{2}\right) z_{2}+A_{2} K_{2} \omega \\
&-K_{2}\left(C_{2} K_{2} \omega+f_{f}+g_{f} g_{f \delta} \delta(t-D(t))\right), \\
& \widehat{\xi}_{2}= z_{2}+K_{2} \omega, \\
& \widehat{d}_{2}= C_{2} \widehat{\xi}_{2} .
\end{aligned}
$$

Here, for simplicity, the disturbances in the attitude angles dynamic equation and in the angle rate dynamic equation are assumed to come from the same exosystem. Then, the virtual controller and actual controller are presented as below for the nondelayed system:

$$
\boldsymbol{\omega}_{d}=\mathbf{g}_{s}^{-1}\left(-c_{s} \mathbf{e}_{s}-\mathbf{f}_{s}-\hat{d}+\dot{\boldsymbol{\Omega}}_{d}\right)-c c_{a_{1}} g_{s}^{-1} \mathbf{e}_{s},
$$

where $\mathbf{e}_{s}=\boldsymbol{\Omega}-\boldsymbol{\Omega}_{d}$ and

$$
\begin{aligned}
\delta_{c} & =g_{2}^{-1}\left(-c_{f} \mathbf{e}_{f}-\mathbf{f}_{f}-\mathbf{g}_{s}^{T} \mathbf{e}_{s}-\widehat{d}-\tilde{d}\right. \\
& \left.+\frac{\partial \boldsymbol{\omega}_{d}}{\partial \boldsymbol{\Omega}}\left(f_{s}+g_{s} \omega+\widehat{d}+\widetilde{d}\right)+\frac{\partial \boldsymbol{\omega}_{d}}{\partial \dot{\boldsymbol{\Omega}}_{d}} \boldsymbol{\Omega}_{d}^{(2)}+\frac{\partial \boldsymbol{\omega}_{d}}{\partial \widehat{d}_{1}} \dot{\vec{d}}\right) \\
& -\cdots-\left(c_{a_{2}} g_{2}^{T}\right. \\
& \left.+c c_{a_{2}} g_{2}^{-1}\left(I-\frac{\partial \boldsymbol{\omega}_{d}}{\partial \boldsymbol{\Omega}}\right)\left(I-\frac{\partial \boldsymbol{\omega}_{d}}{\partial \boldsymbol{\Omega}}\right)^{T}\right) \mathbf{e}_{f}^{T},
\end{aligned}
$$

where $\mathbf{g}_{2} \triangleq \mathbf{g}_{f} \mathcal{g}_{f, \delta}$ and $\mathbf{e}_{f}=\boldsymbol{\omega}-\boldsymbol{\omega}_{d}$.

Finally, the actual controller for the delay system is obtained as

$$
\delta_{c}\left(\bar{\Omega}_{d}^{(2)}, P(t), \overline{\hat{d}}^{(1)}\right),
$$

where the time delay compensation term $P(t)$ is given by (49).

5.2. Simulation. In the Simulink simulation, the mass and moments of inertia are assumed to be constant. Other parameters are set as follows: the flight initial velocity is $2200 \mathrm{~m} / \mathrm{s}$, the thrust is always $400 \mathrm{KN}$, the initial flight height is $27 \mathrm{~km}$, the initial AOA is $0.5^{\circ}$, the initial value of the sideslip angle is $0^{\circ}$, the initial value of the angle around the roll speed is $\mu_{0}=-0.1^{\circ}$, and the elements of the angular velocity in the body frame are all zero. The time delay model is $D(t)=(1+t) / 8(1+2 t)$, which can be easily 


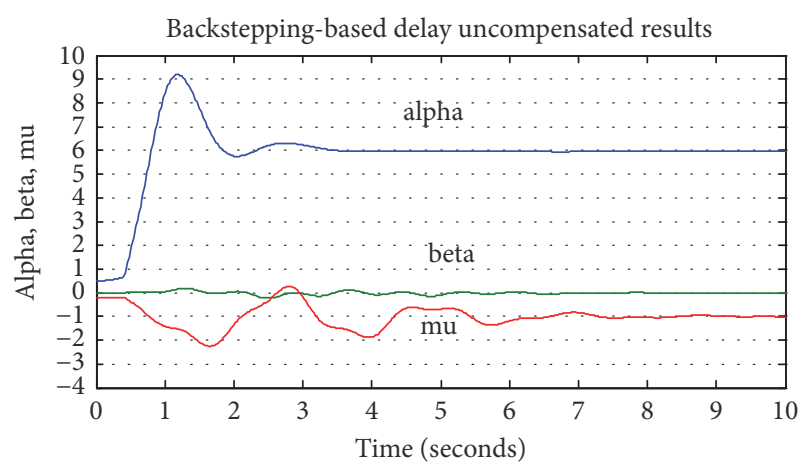

FIGURE 1: Uncompensated tracking results.

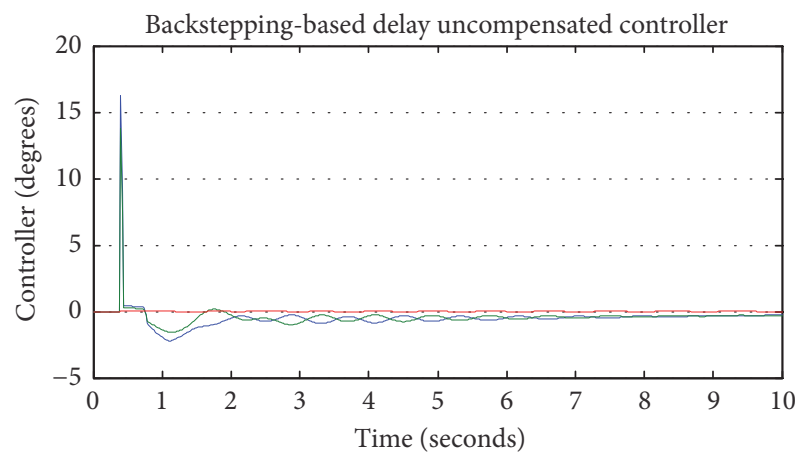

FIgURE 2: Uncompensated actuator deflections.

verified fulfilling Assumption 3. The angles command is $\alpha=$ $6^{\circ} /(s+2)$ or a sine signal with the amplitude of $6^{\circ}, \beta=$ $0^{\circ}$, and $\mu=-1^{\circ}$. The disturbances are given by model (2) with $A=[0,1,1,1 ;-1,0,1,1 ;-1,-1,0,1 ;-1,-1,-1,0]$ and $C=[1,0,0,0 ; 0,1,0,0 ; 0,0,1,0]$ and the initial condition is $0.01 \times[1 ; 1 ; 1 ; 1]$. The simulation results are shown below.

5.2.1. In the Case of Being Disturbance-Free. Firstly, the disturbances are not put into the system, so the time delay compensation control method itself can be verified. When the controller obtained from the nondelayed system is used in the delayed system, the tracking results and actuators' responses are shown in Figures 1 and 2. It is shown that when time delay is small, without delay compensation, the attitude still can track the commands, but there are a big overshoot in the AOA tracking and an apparent oscillation in the tracking error of the angle around the roll speed, which are harmful for the actual flight control. When the delay compensation controller with the same control gains is applied, the tracking results and actuators' response are shown in Figures 3 and 4. It is shown that, using delay compensation, the overshoot in the AOA tracking is quite small, and the oscillation in the tracking error of the angle around the roll speed is effectively suppressed. Those results demonstrate that, under small time delays in the input channel, the delay compensation can significantly improve the control performance and tracking accuracy. In both situations, the actuator deflection angles are quite big, which shows the inherent character of low aerodynamic efficiencies for an AHV's actuation surfaces.

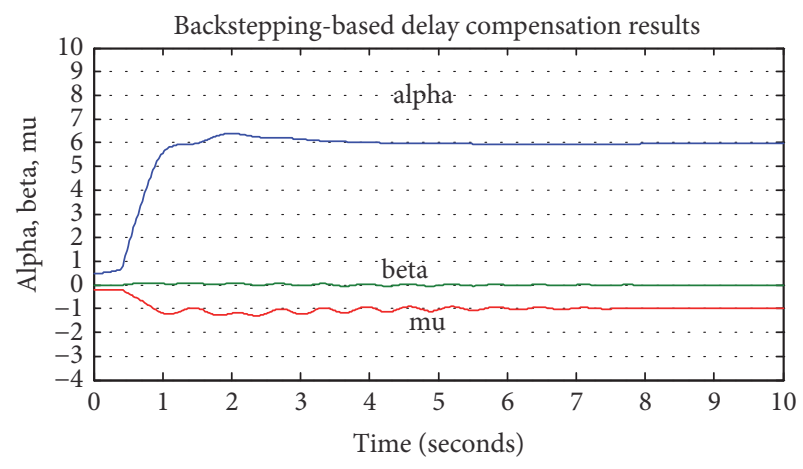

FIGURE 3: Compensated tracking results.

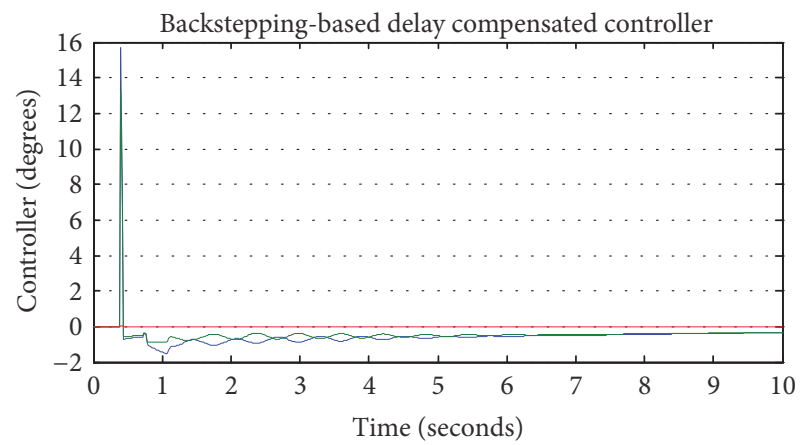

Figure 4: Compensated actuator deflections.

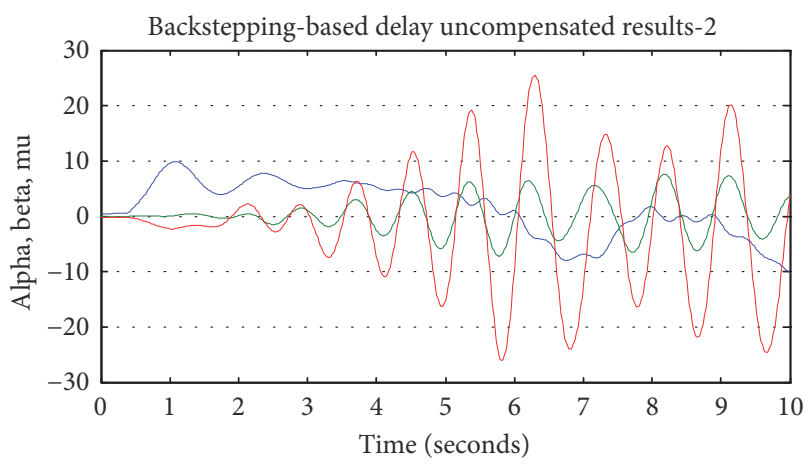

FIGURE 5: Uncompensated tracking results in extreme.

When the time delay is bigger enough, extreme situations come out. In those situations, without delay compensation, the attitude tracking is out of control, as shown in Figures 5 and 6 . Then, using the delay compensation controller with the same control gains, it is shown that the attitude tracking objects are well achieved, as shown in Figures 7 and 8.

A result of AOA tracking to a sinus signal is given in Figures 9 and 10.

The simulation results shown above verify the effectiveness of input delay compensation controller for an AHV attitude tracking control. The compensation control presented in this paper can improve the control performance and tracking accuracy and enhance the stability of the system even in the situations of big input delays. 


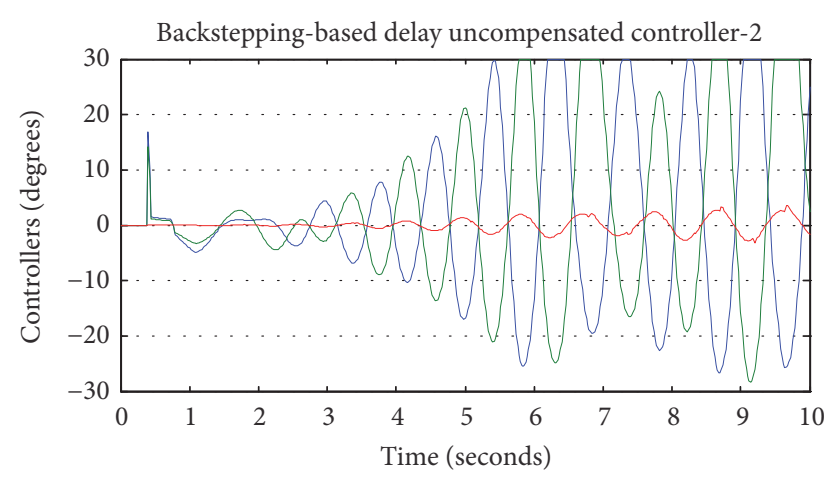

FIGURE 6: Uncompensated actuator deflections in extreme.

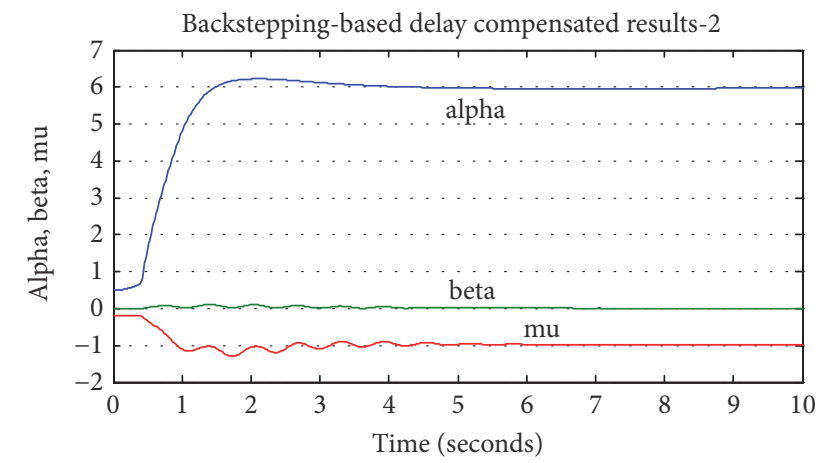

FIGURE 7: Compensated tracking results in extreme.

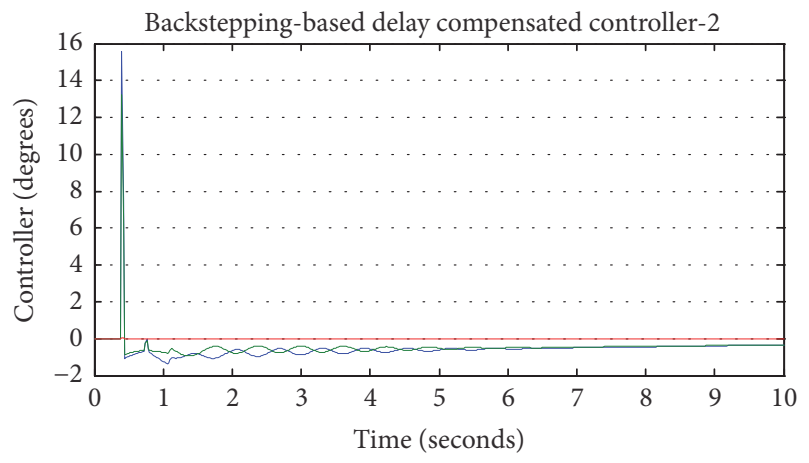

FIGURE 8: Compensated actuator deflections in extreme.

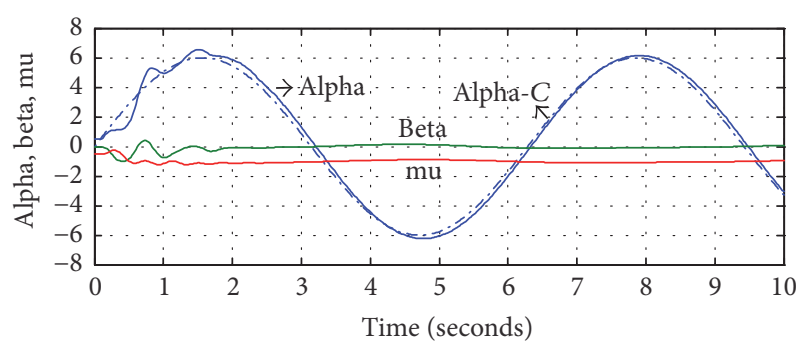

FIGURE 9: Compensated tracking results of AOA sinus.

5.2.2. In the Case of Being Time-Delayed and Disturbed. Then, the disturbances are put into the system, so the whole control method with time delay compensation and disturbance estimation can be verified. Here, a smaller time delay is given as $D(t)=(1+t) / 10(1+2 t)$. When the delay compensation controller with disturbance estimation is applied, the tracking

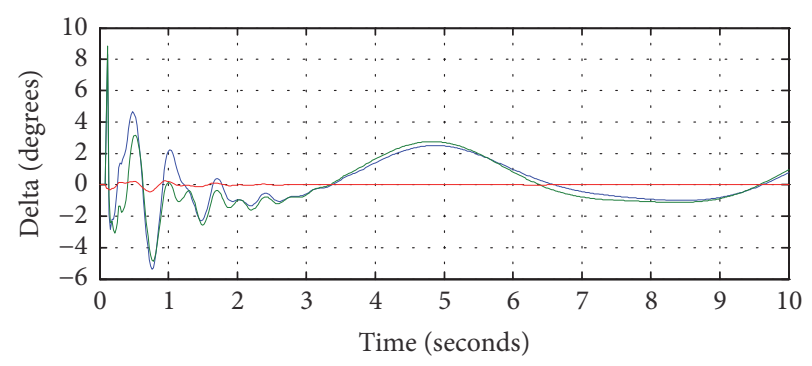

Figure 10: Actuator deflections in AOA tracking of a sinus signal.

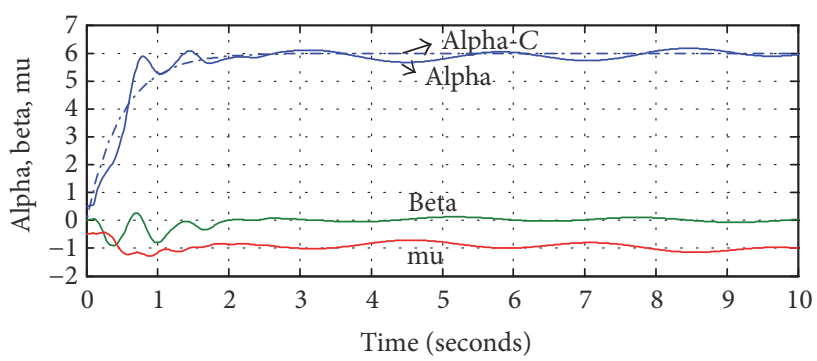

FIGURE 11: Compensated tracking results.

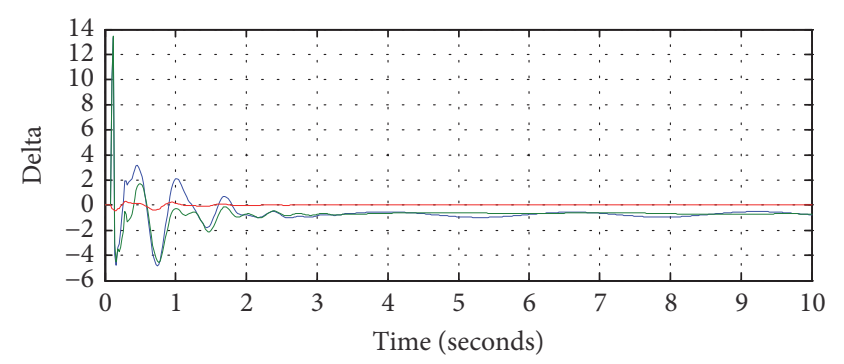

Figure 12: Compensated actuator deflections.

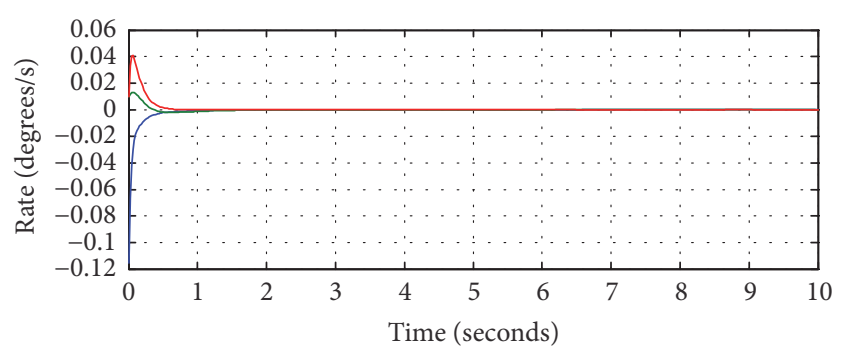

FIGURE 13: Disturbance observer errors in angles dynamic.

results and actuators' response are shown in Figures 11 and 12. The disturbance observers' observing errors are shown in Figures 13 and 14. It is shown that when the disturbances and input delay are considered, the observers in the form of (52) and (53) can asymptotically estimate the disturbance produced by the exosystem, and the attitude commands can be well tracked. In Figure 11, it can be found that there are quite small steady-state errors which are bigger than those in the case of being disturbance-free. The reason for that lies in the adopted Assumption 2. In order to assure the availability of input delay compensation method, the controlled system must be strongly forward complete, which 


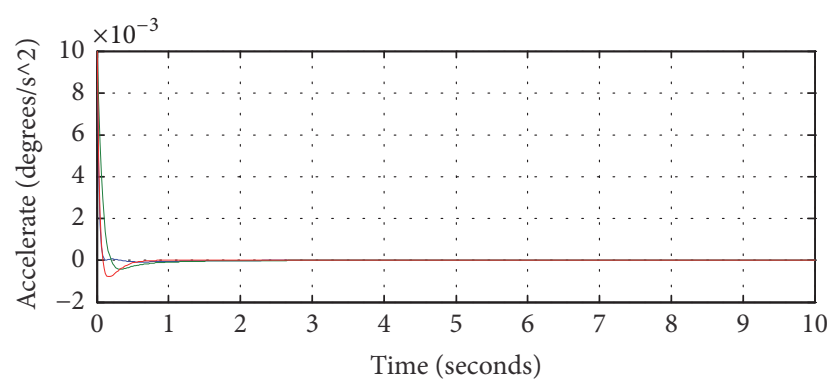

FIgURE 14: Disturbance observer errors in angle_rate dynamic.

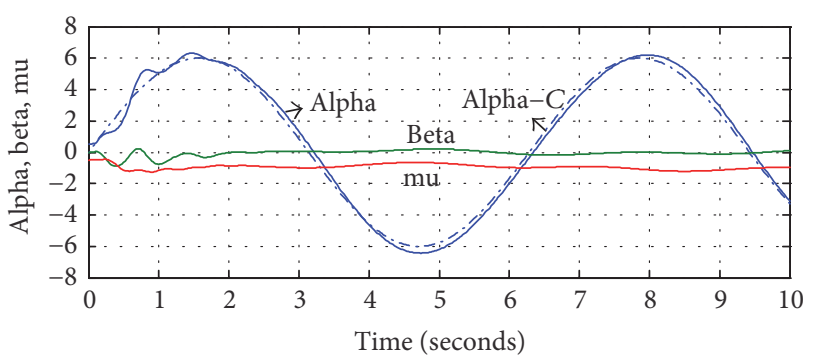

FIGURE 15: Compensated tracking results of AOA sinus.

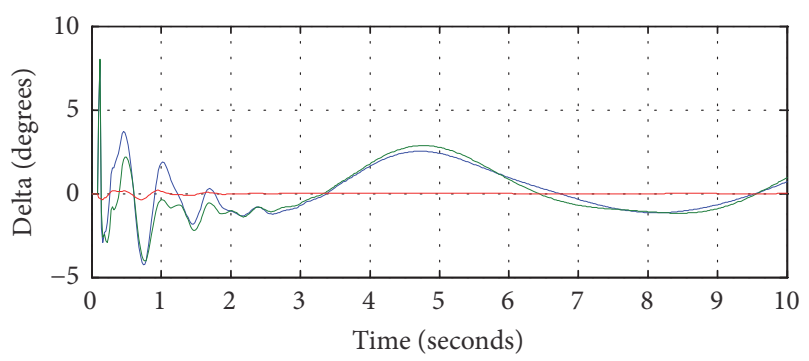

FIgURE 16: Actuator deflections in AOA tracking of a sinus signal.

means the states of the controlled object must be in a safe range all the time. For flight systems, it is reasonable to assume that the attitude tracking error system's states will not get out of the safe range when the input time delay and the disturbance are small enough. So, in the case of being time-delayed and disturbed, the time delay is chosen a little smaller and the disturbance's amplitude is relatively small. Actually, the disturbance's amplitude (about $0.01 \mathrm{rad} / \mathrm{s}$ ) is not quite small with respect to the speed of $\mathrm{AOA}^{\prime}$ tracking (about $0.05 \mathrm{rad} / \mathrm{s}$ ). Although there are steady-state errors in the attitude tracking, it is shown in Figure 15 that the AOA can efficiently track a complex signal, such as a sine signal. All the actuator deflection results in Figures 12 and 16 indicate that the control method used here can make the AHV's attitude tracking realized without reaching the deflecting constraint of 30 degrees.

\section{Conclusion}

The main contribution in this article is the new framework of solving a nonlinear input delay regulation problem. It is impossible to get an analytic solution when the nonlinear regulation equations contain delay terms, so the nonlinear regulation problem with input delay has not been solved for a long time. In this paper, based on the framework we proposed, the nonlinear regulation problem with input delay is settled for the first time, for the class of strict feedback nonlinear systems. The framework proposed consisted of nonlinear damping controllers, nonlinear disturbance observers, and a nonlinear time delay predictor-based compensator, under the proved separation principle. Then, in application, the attitude tracking control of an AHV with considering the input delay is studied for the first time, using the proposed framework. The input delay is abstracted from the controller signal transmission and actuator dynamics and the disturbances come from the wind or modeling error. Based on the simulation results, we have demonstrated the advantage of the control method described in this paper and its availability for AHVs' attitude control.

\section{Conflicts of Interest}

The authors declare that there are no conflicts of interest.

\section{Acknowledgments}

This paper is supported by National Natural Science Foundation of China (no. 61374212).

\section{References}

[1] A. Isidori, Nonlinear Control Systems, Communications and Control Engineering Series, Springer, Berlin, Germany, 3rd edition, 1995.

[2] C. I. Byrnes and A. Isidori, "Output regulation for nonlinear systems: an overview," International Journal of Robust and Nonlinear Control, vol. 10, no. 5, pp. 323-337, 2000.

[3] B. A. Francis, "The linear multivariable regulator problem," SIAM Journal on Control and Optimization, vol. 15, no. 3, pp. 486-505, 1977.

[4] A. Isidori and C. I. Byrnes, "Output regulation of nonlinear systems," IEEE Transactions on Automatic Control, vol. 35, no. 2, pp. 131-140, 1990.

[5] J. Huang and Z. Chen, "A general framework for tackling the output regulation problem," IEEE Transactions on Automatic Control, vol. 49, no. 12, pp. 2203-2218, 2004.

[6] A. Pavlov, N. van de Wouw, and H. Nijmeijer, Uniform Output Regulation of Nonlinear Systems: A Convergent Dynamics Approach, Birkhäuser, Boston, Mass, USA, 2005.

[7] T.-J. Tarn, P. Sanposh, D. Cheng, and M. Zhang, "Output regulation for nonlinear systems: some recent theoretical and experimental results," IEEE Transactions on Control Systems Technology, vol. 13, no. 4, pp. 605-610, 2005.

[8] C. I. Byrnes, F. Delli Priscoli, A. Isidori, and W. Kang, "Structurally stable output regulation of nonlinear systems," Automatica, vol. 33, no. 3, pp. 369-385, 1997.

[9] H. K. Khalil, "On the design of robust servomechanisms for minimum phase nonlinear systems," International Journal of Robust and Nonlinear Control, vol. 10, no. 5, pp. 339-361, 2000.

[10] A. Serrani and A. Isidori, "Global robust output regulation for a class of nonlinear systems," Systems and Control Letters, vol. 39, no. 2, pp. 133-139, 2000. 
[11] C. I. Byrnes and A. Isidori, "Limit sets, zero dynamics, and internal models in the problem of nonlinear output regulation," IEEE Transactions on Automatic Control, vol. 48, no. 10, pp. 1712-1723, 2003.

[12] Y. Hong, X. Wang, and Z.-P. Jiang, "Distributed output regulation of leader-follower multi-agent systems," International Journal of Robust and Nonlinear Control, vol. 23, no. 1, pp. 4866, 2013.

[13] Y. Su, Y. Hong, and J. Huang, "A general result on the robust cooperative output regulation for linear uncertain multi-agent systems," IEEE Transactions on Automatic Control, vol. 58, no. 5, pp. 1275-1279, 2013.

[14] C. I. Byrnes and A. Isidori, "Nonlinear internal models for output regulation," IEEE Transactions on Automatic Control, vol. 49, no. 12, pp. 2244-2247, 2004.

[15] Z. T. Ding, "Global stabilization and disturbance suppression of a class of nonlinear systems with uncertain internal model," Automatica, vol. 39, no. 3, pp. 471-479, 2003.

[16] R. Marino, G. L. Santosuosso, and P. Tomei, "Robust adaptive compensation of biased sinusoidal disturbances with unknown frequency," Automatica, vol. 39, no. 10, pp. 1755-1761, 2003.

[17] D. Xu and J. Huang, "Robust adaptive control of a class of nonlinear systems and its applications," IEEE Transactions on Circuits and Systems. I. Regular Papers, vol. 57, no. 3, pp. 691702, 2010.

[18] Z. Chen and J. Huang, "Dissipativity, stabilization, and regulation of cascade-connected systems," IEEE Transactions on Automatic Control, vol. 49, no. 5, pp. 635-650, 2004.

[19] Z. Ding, "Universal disturbance rejection for nonlinear systems in output feedback form," IEEE Transactions on Automatic Control, vol. 48, no. 7, pp. 1222-1227, 2003.

[20] R. D. Nussbaum, "Some remarks on a conjecture in parameter adaptive control," Systems and Control Letters, vol. 3, no. 5, pp. 243-246, 1983.

[21] L. Liu and J. Huang, "Global robust output regulation of lower triangular systems with unknown control direction," Automatica, vol. 44, no. 5, pp. 1278-1284, 2008.

[22] L. Liu, Z. Chen, and J. Huang, "Parameter convergence and minimal internal model with an adaptive output regulation problem," Automatica, vol. 45, no. 5, pp. 1306-1311, 2009.

[23] K. Gu, V. L. Kharitonov, and J. Chen, Stability of Time-Delay Systems, Control Engineering, Birkhauser, Boston, Mass, USA, 2003.

[24] E. Fridman, Introduction to Time-Delay Systems, Birkhäuser, 2014.

[25] J. Chen and H. A. Latchman, "Frequency sweeping tests for stability independent of delay," IEEE Transactions on Automatic Control, vol. 40, no. 9, pp. 1640-1645, 1995.

[26] X. Li and C. E. De Souza, "Delay-dependent robust stability and stabilization of uncertain linear delay systems: a linear matrix inequality approach," IEEE Transactions on Automatic Control, vol. 42, no. 8, pp. 1144-1148, 1997.

[27] N. N. Krasovskii, "On the applications of the second Lyapunov method for equations with delay," Journal of Applied Mathematics and Mechanics, vol. 20, pp. 315-327, 1956.

[28] B. S. Razumihin, "The application of Lyapunov's method to problems in the stability of systems with delay," Automation and Remote Control, vol. 21, pp. 515-520, 1960.

[29] Y. He, M. Wu, J.-H. She, and G.-P. Liu, "Parameter-dependent Lyapunov functional for stability of time-delay systems with polytopic-type uncertainties," IEEE Transactions on Automatic Control, vol. 49, no. 5, pp. 828-832, 2004.
[30] E. Fridman, "Descriptor discretized Lyapunov functional method: analysis and design," IEEE Transactions on Automatic Control, vol. 51, no. 5, pp. 890-897, 2006.

[31] P. Park, "A delay-dependent stability criterion for systems with uncertain time-invariant delays," IEEE Transactions on Automatic Control, vol. 44, no. 4, pp. 876-877, 1999.

[32] Y. S. Moon, P. Park, and W. H. Kwon, "Robust stabilization of uncertain input-delayed systems using reduction method," Automatica, vol. 37, no. 2, pp. 307-312, 2001.

[33] J.-H. Kim, "Further improvement of Jensen inequality and application to stability of time-delayed systems," Automatica, vol. 64, pp. 121-125, 2016.

[34] M. Jankovic, "Cross-term forwarding for systems with time delay," IEEE Transactions on Automatic Control, vol. 54, no. 3, pp. 498-511, 2009.

[35] F. Mazenc and P.-A. Bliman, "Backstepping design for timedelay nonlinear systems," IEEE Transactions on Automatic Control, vol. 51, no. 1, pp. 149-154, 2006.

[36] P. Pepe and Z.-P. Jiang, "A Lyapunov-Krasovskii methodology for ISS and iISS of time-delay systems," Systems and Control Letters, vol. 55, no. 12, pp. 1006-1014, 2006.

[37] P. Pepe, I. Karafyllis, and Z.-P. Jiang, "On the LiapunovKrasovskii methodology for the ISS of systems described by coupled delay differential and difference equations," Automatica, vol. 44, no. 9, pp. 2266-2273, 2008.

[38] F. Mazenc and M. Malisoff, "Stabilization of a chemostat model with Haldane growth functions and a delay in the measurements," Automatica, vol. 46, no. 9, pp. 1428-1436, 2010.

[39] F. Mazenc and S.-I. Niculescu, "Generating positive and stable solutions through delayed state feedback," Automatica, vol. 47, no. 3, pp. 525-533, 2011.

[40] F. Mazenc, S.-I. Niculescu, and M. Bekaik, "Backstepping for nonlinear systems with delay in the input revisited," SIAM Journal on Control and Optimization, vol. 49, no. 6, pp. 22632278, 2011.

[41] N. Bekiaris-Liberis and M. Krstic, "Compensation of timevarying input and state delays for nonlinear systems," Journal of Dynamic Systems Measurement and Control, vol. 134, no. 1, pp. 335-339, 2012.

[42] N. Bekiaris-Liberis and M. Krstic, "Compensation of statedependent input delay for nonlinear systems," IEEE Transactions on Automatic Control, vol. 58, no. 2, pp. 275-289, 2013.

[43] M. Krstic, "Input delay compensation for forward complete and strict-feedforward nonlinear systems," IEEE Transactions on Automatic Control, vol. 55, no. 2, pp. 287-303, 2010.

[44] M. Krstic, "Lyapunov stability of linear predictor feedback for time-varying input delay," IEEE Transactions on Automatic Control, vol. 55, no. 2, pp. 554-559, 2010.

[45] I. Karafyllis, "Stabilization by means of approximate predictors for systems with delayed input," SIAM Journal on Control and Optimization, vol. 49, no. 3, pp. 1100-1123, 2011.

[46] N. Bekiaris-Liberis and M. Krstic, "Robustness of nonlinear predictor feedback laws to time- and state-dependent delay perturbations," Automatica, vol. 49, no. 6, pp. 1576-1590, 2013.

[47] L. Marconi, L. Praly, and A. Isidori, "Output stabilization via nonlinear Luenberger observers," SIAM Journal on Control and Optimization, vol. 45, no. 6, pp. 2277-2298, 2007.

[48] F. D. Priscoli, L. Marconi, and A. Isidori, "Adaptive observers as nonlinear internal models," Systems \& Control Letters, vol. 55, no. 8, pp. 640-649, 2006. 
[49] C. I. Byrnes, I. G. Lauko, D. Gilliam, and V. I. Shubov, "Output regulation for linear distributed parameter systems," IEEE Transactions on Automatic Control, vol. 45, no. 12, pp. 22362252, 2000.

[50] E. Fridman, "Output regulation of nonlinear systems with delay," Systems and Control Letters, vol. 50, no. 2, pp. 81-93, 2003.

[51] H. Xu, M. D. Mirmirani, and P. A. Ioannou, "Adaptive sliding mode control design for a hypersonic flight vehicle," Journal of Guidance, Control, and Dynamics, vol. 27, no. 5, pp. 829-838, 2004.

[52] L. Fiorentini, A. Serrani, M. A. Bolender, and D. B. Doman, "Nonlinear robust adaptive control of flexible air-breathing hypersonic vehicles," Journal of Guidance, Control, and Dynamics, vol. 32, no. 2, pp. 401-416, 2009.

[53] W.-H. Chen, "Disturbance observer based control for nonlinear systems," IEEE/ASME Transactions on Mechatronics, vol. 9, no. 4, pp. 706-710, 2004.

[54] J. D. Shaughnessy, S. Z. Pinckney, J. D. McMinn et al., "Hypersonic vehicle simulation model: winged-cone configuration," NASA Technical Memorandum 102610, 1990. 


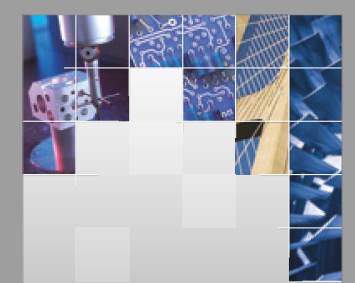

\section{Enfincering}
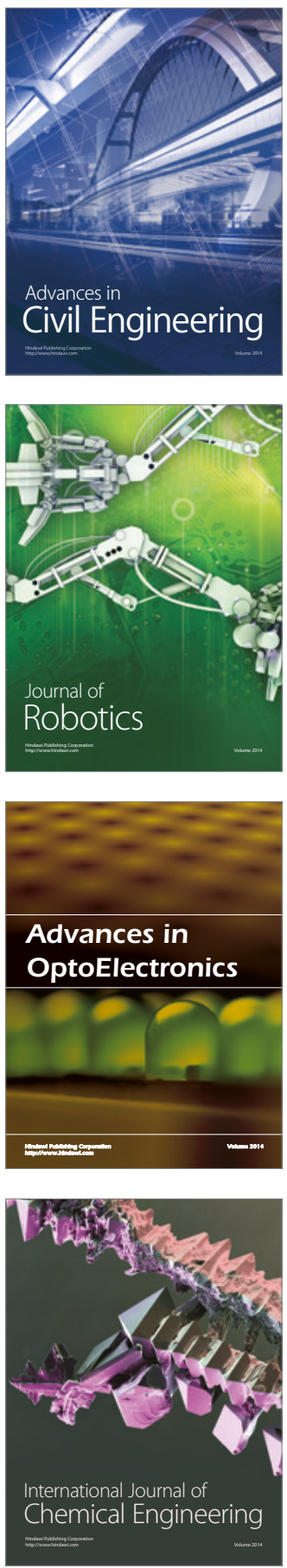

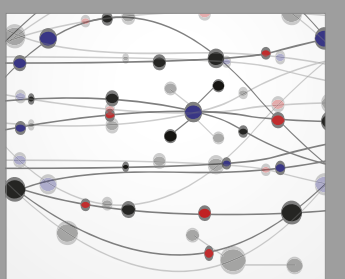

The Scientific World Journal

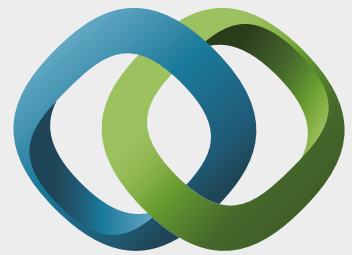

\section{Hindawi}

Submit your manuscripts at

https://www.hindawi.com
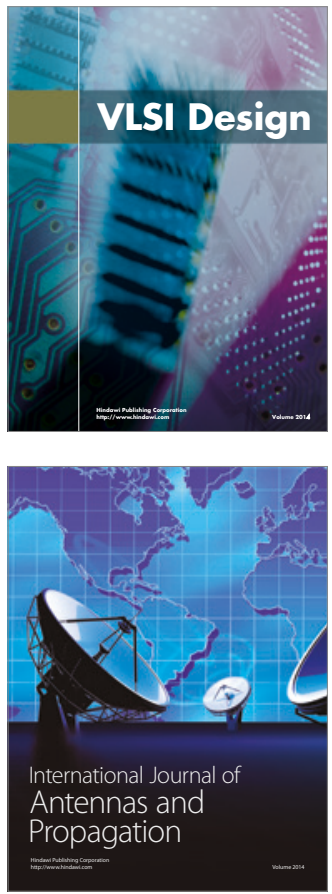

\section{Rotating}

Machinery
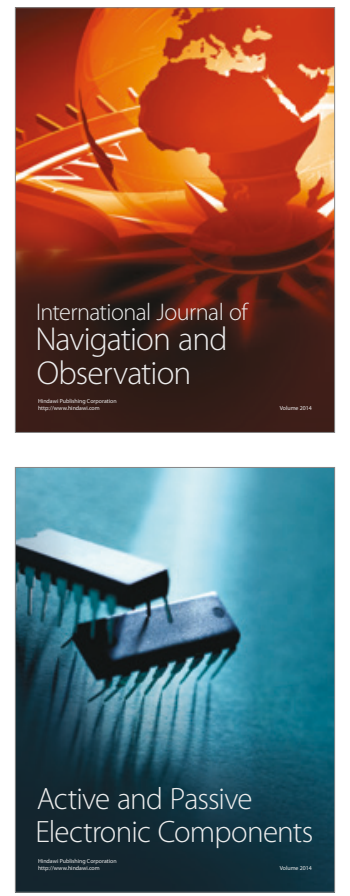
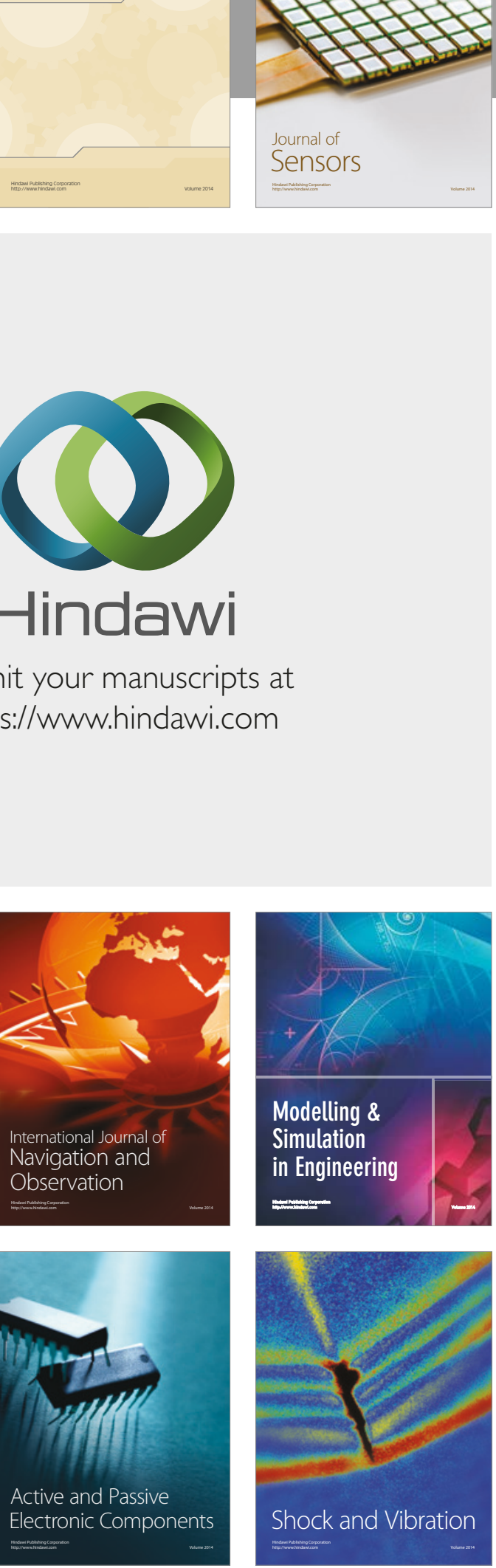
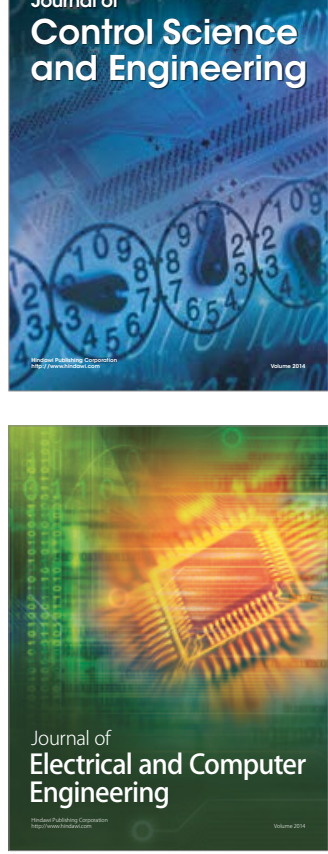

Distributed

Journal of

Control Science

and Engineering
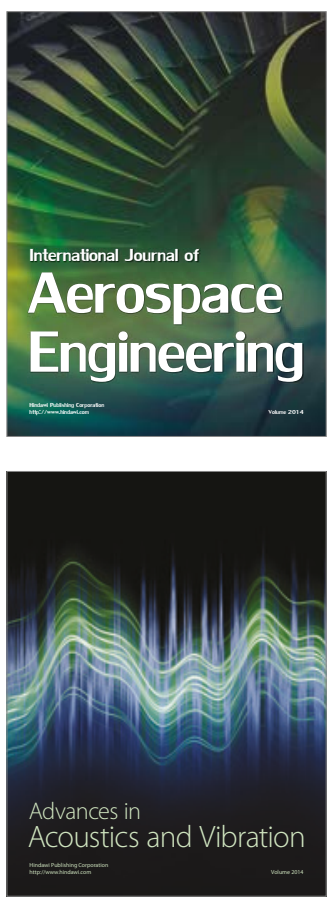

Sensor Networks 